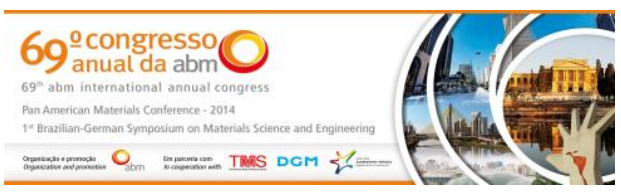

Tema: Produtos metálicos ferrosos

\title{
O EFEITO DA INDUÇÃO MÁXIMA SOBRE A ENERGIA DISSIPADA POR HISTERESE EM AÇOS ELÉTRICOS DE GRÃO NÃO ORIENTADO*
}

\section{Resumo}

Daniel Luiz Rodrigues Júnior ${ }^{1}$ Fernando José Gomes Landgraf ${ }^{2}$ Sérgio Antônio Romero ${ }^{3}$

O presente trabalho avalia o efeito da indução máxima sobre a energia dissipada por histerese magnética em amostras de aços elétricos de grão não orientado. Tal relação foi descrita por Steinmetz como uma lei de potência cujo expoente tem valor 1,6. No entanto, esta lei falha quando aplicada a uma ampla faixa de induções magnéticas. Com o objetivo de se avaliar a aplicabilidade dessa equação chapas de aço elétrico foram caracterizadas. Ciclos de histerese foram extraídos com indução máxima variando entre 0,4 e 2,2 T com frequência $5 \mathrm{mHz}$. A área no interior dos ciclos corresponde à energia dissipada. Estes resultados mostraram uma diminuição do valor do expoente para induções entre 1,2 e 1,6 T. Este fato pode ser explicado pela estagnação do valor do campo coercivo nessa faixa de dados.

Palavras-chave: Aço elétrico; Indução máxima; Perda magnética; Magnetização.

\section{THE EFFECT OF THE MAXIMUM INDUCTION ON THE ENERGY DISSIPATED BY HYSTERESIS IN NON ORIENTED ELECTRICAL STEEL}

\section{Abstract}

The present work evaluates the effect of maximum induction on the hysteresis loss in non oriented electrical steel samples. This relation was described by Steinmetz as a power law whose the exponent has value 1.6. However, this law fails when applied to a wide range of magnetic inductions. In order to evaluate the applicability of this equation, electrical steel sheets were characterized. Hysteresis loops are extracted with maximum induction ranging from 0.4 up to $2.2 \mathrm{~T}$ at frequency $5 \mathrm{mHz}$. The area enclosed by the loop corresponds to the energy dissipated. These results showed a decreasing of the exponent value to inductions starting from 1.2 to $1.6 \mathrm{~T}$. This fact can be explained by a stagnation of the coercive field at this range of data.

Keywords: Electrical steel; Maximum induction; Magnetic loss; Magnetization.

1 Mestre em Engenharia de Materiais, doutorando, Departamento de Engenharia Metalúrgica e de Materiais da Escola Politécnica, Universidade São Paulo (USP), São Paulo, SP, Brasil.

2 Professor Doutor, Departamento de Engenharia Metalúrgica e de Materiais da Escola Politécnica, Universidade São Paulo, Diretor Presidente do Instituto de Pesquisas Tecnológicas, São Paulo, $S P$, Brasil.

3 Doutor em Engenharia de Materiais, servidor, Instituto de Física, USP, São Paulo, SP, Brasil.

* Contribuição técnica ao 69 Congresso Anual da ABM - Internacional e ao 14ํㅡㄹ ENET - Encontro Nacional de Estudantes de Engenharia Metalúrgica, de Materiais e de Minas, 21 a 25 de julho de 2014, São Paulo, SP, Brasil. 


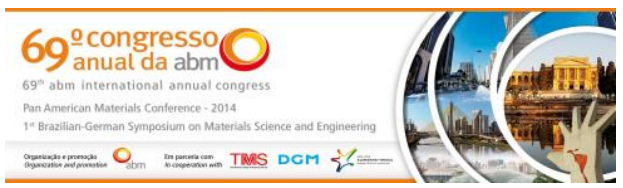

\section{INTRODUÇÃO}

O aço elétrico de grão não orientado é a principal matéria prima usada na construção de máquinas elétricas tais como motores e transformadores. Nestes dispositivos, o aço elétrico atua como condutor de fluxo magnético. Uma das principais características dessa classe de materiais é a chamada perda magnética. Tal perda consiste na energia dissipada ao longo dos ciclos de magnetização e desmagnetização típicos de sua aplicação. A necessidade de produção de dispositivos energeticamente mais eficientes impõe redução da perda magnética em máquinas elétricas.

O desempenho desses aços é fruto de características microestruturais [1] e de parâmetros operacionais tais como frequência de excitação [2] e indução máxima sendo que esta última teve seu efeito descrito ainda em 1892 por Steinmetz [3]. Em seu trabalho, o autor relaciona a energia dissipada por histerese e a indução máxima através de uma lei de potência (Equação 1).

$$
W_{h}=k \times B_{\max }^{1,6}
$$

onde é "k" uma constante e $B_{\max }$ é a indução máxima.

No entanto, para valores de indução máxima superiores a 1,2 T o expoente da indução máxima não se mantém [4]. Tal limitação impede uma maior precisão na previsão do comportamento de motores elétricos quando submetidos à indução máxima que exceda o valor usual de $1.5 \mathrm{~T}$. $\mathrm{O}$ presente trabalho avalia 0 comportamento da energia dissipada para uma ampla faixa de valores de indução máxima. A energia dissipada foi medida através da área de ciclos de histerese magnética para diferentes valores de indução máxima. Os dados foram comparados ao comportamento previsto pela lei de Steinmetz. Também foram avaliados os mecanismos de magnetização que atuam ao longo da excitação magnética.

\section{MATERIAIS E MÉTODOS}

Para a condução desse trabalho o material sob estudo foi extraído de uma bobina de aço elétrico de grão não orientado, laminado a frio, cuja composição é dada pela Tabela, espessura $0,5 \mathrm{~mm}$, densidade $7781 \mathrm{~kg} / \mathrm{m}^{3}$ e $27,62 \mu \Omega . \mathrm{cm}$ de resistividade elétrica.

Tabela 1: Composição química do material sob estudo.

\begin{tabular}{ccccccccccc}
\hline \hline $\mathrm{C}$ & $\mathrm{Mn}$ & $\mathrm{P}$ & $\mathrm{S}$ & $\mathrm{Si}$ & $\mathrm{Al}$ & $\mathrm{Cu}$ & $\mathrm{Cr}$ & $\mathrm{Ni}$ & $\mathrm{Mo}$ & $\mathrm{Ti}$ \\
\hline 0.001 & 0.524 & 0.0374 & 0.0075 & 0.819 & 0.215 & 0.008 & 0.016 & 0.005 & 0.0011 & 0.0025 \\
\hline \hline
\end{tabular}

Medidas magnéticas foram feitas em quadro de Epstein usando-se um conjunto de amostras composto por doze chapas. A aplicação do campo magnético se deu paralela à direção de laminação. A dissipação de energia foi calculada a partir dos ciclos de histerese e corresponde a área compreendida por essas curvas. A medição de perdas histeréticas em quadro de Epstein se deu em regime quase estático (frequência de $5 \mathrm{mHz}$ ) para induções máximas entre 0,4 e 1,6 T. Este processo de medição fez uso de um fluxímetro Walker MF-3D conectado à bobina secundária do quadro de Epstein e de um resistor shunt conectado a um multímetro HP 34401A.

\footnotetext{
* Contribuição técnica ao 69 Congresso Anual da ABM - Internacional e ao 14ํㅡㄹ ENEMET - Encontro Nacional de Estudantes de Engenharia Metalúrgica, de Materiais e de Minas, 21 a 25 de julho de 2014, São Paulo, SP, Brasil.
} 
A indução máxima tem seu valor determinado pela intensidade do campo magnético aplicado. O quadro de Epstein tem como limite máximo um campo de $6000 \mathrm{~A} / \mathrm{m}$. Diante dessa limitação, tornou-se necessário o uso de um magnetômetro de amostra vibrante (MAV). Esse equipamento executa a caracterização magnética através da medição do momento magnético de uma amostra quando esta é vibrada perpendicularmente a um campo magnético uniforme [5]. $O$ aparelho usado foi fabricado pela EG\&G Princeton Applied Research, modelo 4500. Os corpos de prova foram preparados em formato de pequenos paralelepípedos de dimensões $5 \times 0,5 \times 0,5 \mathrm{~mm}$. O campo aparente foi calculado através da Equação 2, que relaciona os campos aplicado $(\mathrm{H})$ e desmagnetizante $\left(\mathrm{H}_{\mathrm{d}}\right)$. Este último foi determinado através da Equação 3 para fator desmagnetizante $\left(\mathrm{N}_{\mathrm{d}}\right)$ com valor $1,34 \times 10^{-12}[6]$.

$$
\begin{gathered}
H_{a p}=H+H_{d} \\
H_{d}=-N_{d} \times M
\end{gathered}
$$

\section{RESULTADOS E DISCUSSÃO}

A relação entre a energia dissipada por histerese $\left(W_{h}\right)$ e a indução máxima é descrita pela Figura. Nota-se que, para o intervalo de $B_{\max }$ analisado, a relação apresenta exponencial de 1,57, próximo ao previsto pela equação de Steinmetz. No entanto, conforme indica a Figura, há intervalos onde o comportamento desvia do estabelecido pela Equação 1. Para $B_{\max }$ variando entre 1,2 e 1,6 T há uma notável queda no valor do expoente, indo para 1,18. A diminuição desse valor também foi observada por Landgraf [4] e Ball [7]. Contudo, esse valor parece se recuperar indo para 1,51 quando a indução máxima supera 1,7 T.

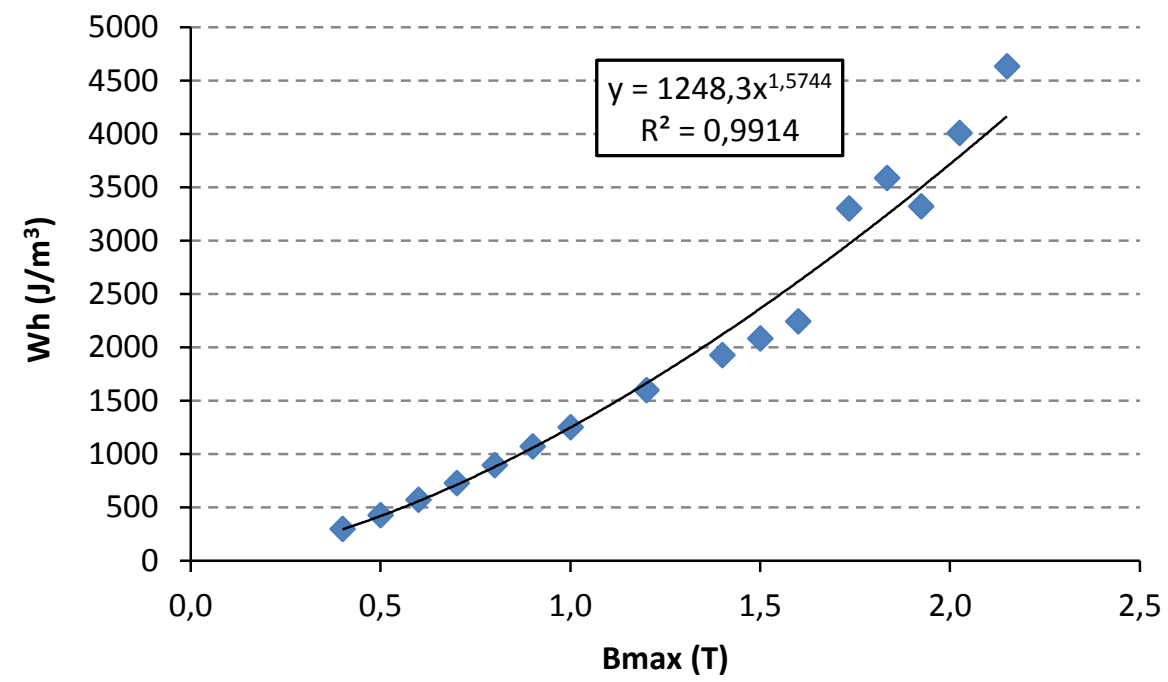

Figura 1: Aumento da energia dissipada pela histerese em função do crescimento da indução máxima.

\footnotetext{
* Contribuição técnica ao 69 Congresso Anual da ABM - Internacional e ao 14ํㅡㄹ ENEMET - Encontro Nacional de Estudantes de Engenharia Metalúrgica, de Materiais e de Minas, 21 a 25 de julho de 2014, São Paulo, SP, Brasil.
} 

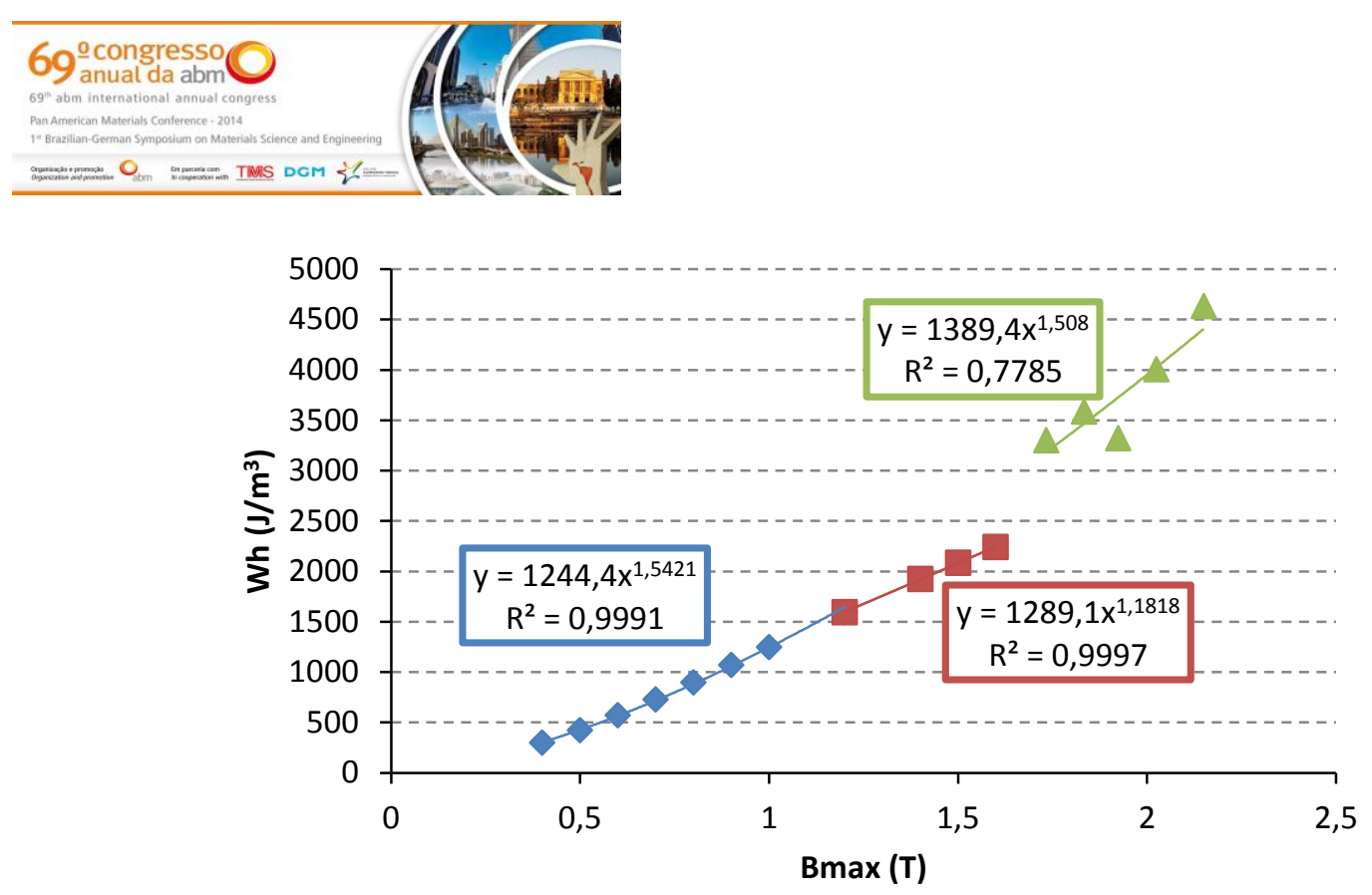

Figura 2: Energia dissipada por histerese em função da indução máxima apresentando os diferentes intervalos de variação de $B_{\max }$ e suas leis de potência.

Uma análise mais detalhada dos ciclos de histereses pode ser feita a partir do procedimento de subdivisão. Este método propõe a separação da histerese em regiões delimitadas pela indução onde a permeabilidade tem seu valor máximo $\left(B_{\mu \max }\right)$. Essas regiões, chamadas de alta e baixa indução, correspondem a faixas de predomínio de mecanismos de magnetização. Na região de baixa indução, ocorre o predomínio da magnetização por movimento de paredes de domínio. Na região de alta indução prevalece a magnetização por rotação de domínios [8].

A Figura relata o comportamento da indução de permeabilidade máxima $\left(B_{\mu \max }\right) \mathrm{com}$ o aumento da indução máxima. Nota-se que, para $B_{\max }$ superior a 1,2 T, os valores de $B_{\mu m a x}$ apresentam apenas pequeno incremento. Assim, compreende-se que a diferença entre os expoentes observados, comparando-se os dois intervalos de $B_{\max }$ descritos na Figura onde indução máxima é superior a 1,2 T, não se pode ser atribuída à mudança da posição do ponto onde ocorre a mudança de predomínio de mecanismo de magnetização.

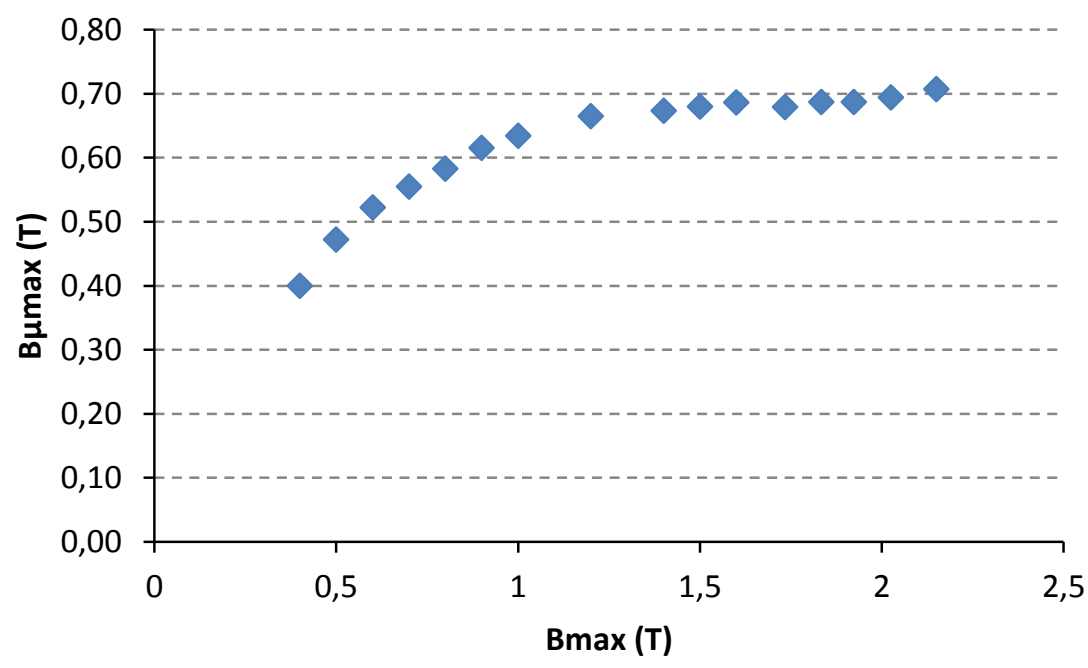

Figura 3: Evolução da entra indução de permeabilidade máxima $\left(\mathrm{B}_{\mu \max }\right)$ com o aumento da indução máxima.

\footnotetext{
* Contribuição técnica ao $69^{\circ}$ Congresso Anual da ABM - Internacional e ao 14ํㅡㄹ ENEMET - Encontro Nacional de Estudantes de Engenharia Metalúrgica, de Materiais e de Minas, 21 a 25 de julho de 2014, São Paulo, SP, Brasil.
} 
A energia dissipada na região de alta indução ( $H$ _Wh) apresenta persistente crescimento com o aumento da indução máxima, conforme mostra a Figura. Dessa forma, esses dados apresentam o mesmo comportamento ao longo de toda a faixa de induções analisadas. Interpretação diferente pode ser feita observando-se a Figura que descreve a evolução da energia dissipada na região de baixa ( $L$ _Wh) indução com o aumento de $B_{\max }$. Assim como no gráfico da Figura, a energia dissipada tem comportamento diferente quando comparamos os intervalos de $B_{\max }$ variando entre 1,2 e 1,6 T com os resultados da parte final do gráfico, ou seja, indução máxima superior a 1,7 T.

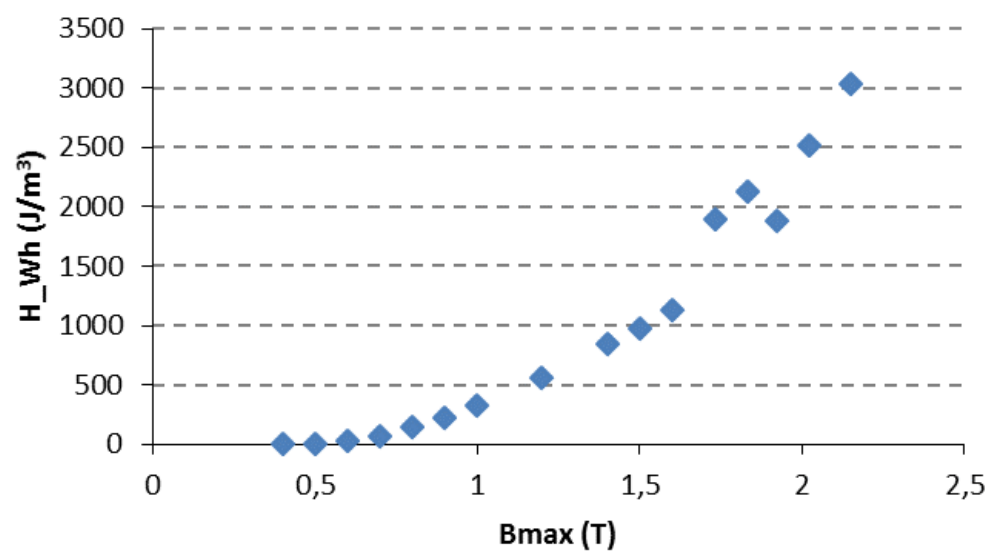

Figura 4: Energia dissipada na região de alta indução em função da indução máxima.

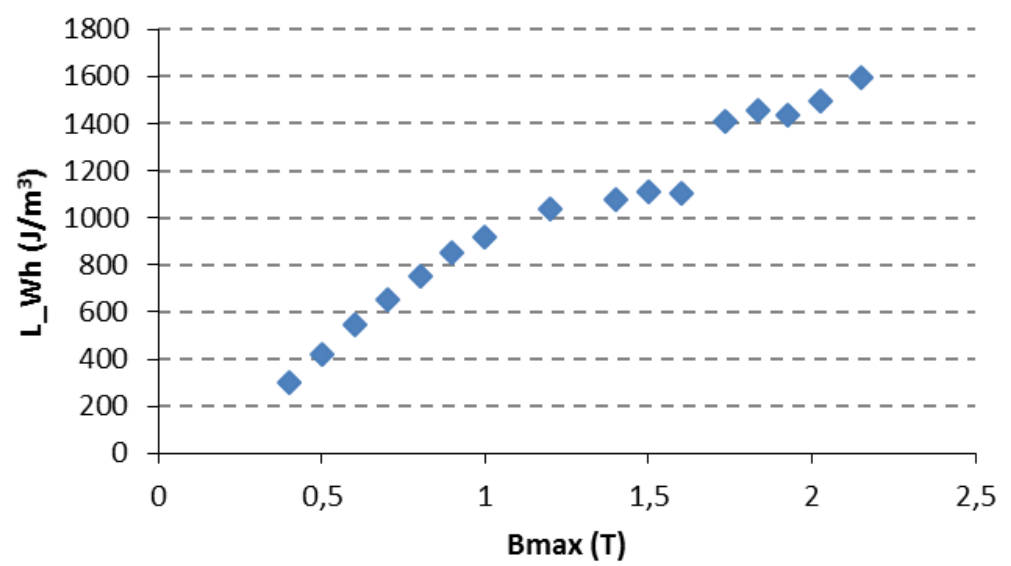

Figura 5: Energia dissipada na região de baixa indução em função da indução máxima.

A Figura demonstra que a evolução do campo coercivo com o aumento de $B_{\max }$ apresenta perfil bastante semelhante ao observado para a perda de baixa indução. Para um intervalo de induções máximas entre 1,2 e 1,6 T, o campo coercivo praticamente não se altera. Isso limita o aumento da área da histerese deixando-o apenas a cargo do aumento da indução máxima. Tal fato provocou a diminuição do expoente da equação de Steinmetz para esse intervalo de dados. Uma razão para essa mudança no comportamento do campo coercivo pode ser atribuída ao método de medição. Para que valores de indução máxima superiores a 1,7 T fossem alcançados utilizou-se um magnetômetro de amostra vibrante. Para os ensaios conduzidos com $\mathrm{B}_{\max }$ superiores a 1,2 $\mathrm{T}$, apenas os resultados produzidos através do MAV apresentaram crescimento do campo coercivo.

\footnotetext{
* Contribuição técnica ao 69 Congresso Anual da ABM - Internacional e ao 14ํㅡㄹ ENEMET - Encontro Nacional de Estudantes de Engenharia Metalúrgica, de Materiais e de Minas, 21 a 25 de julho de 2014, São Paulo, SP, Brasil.
} 

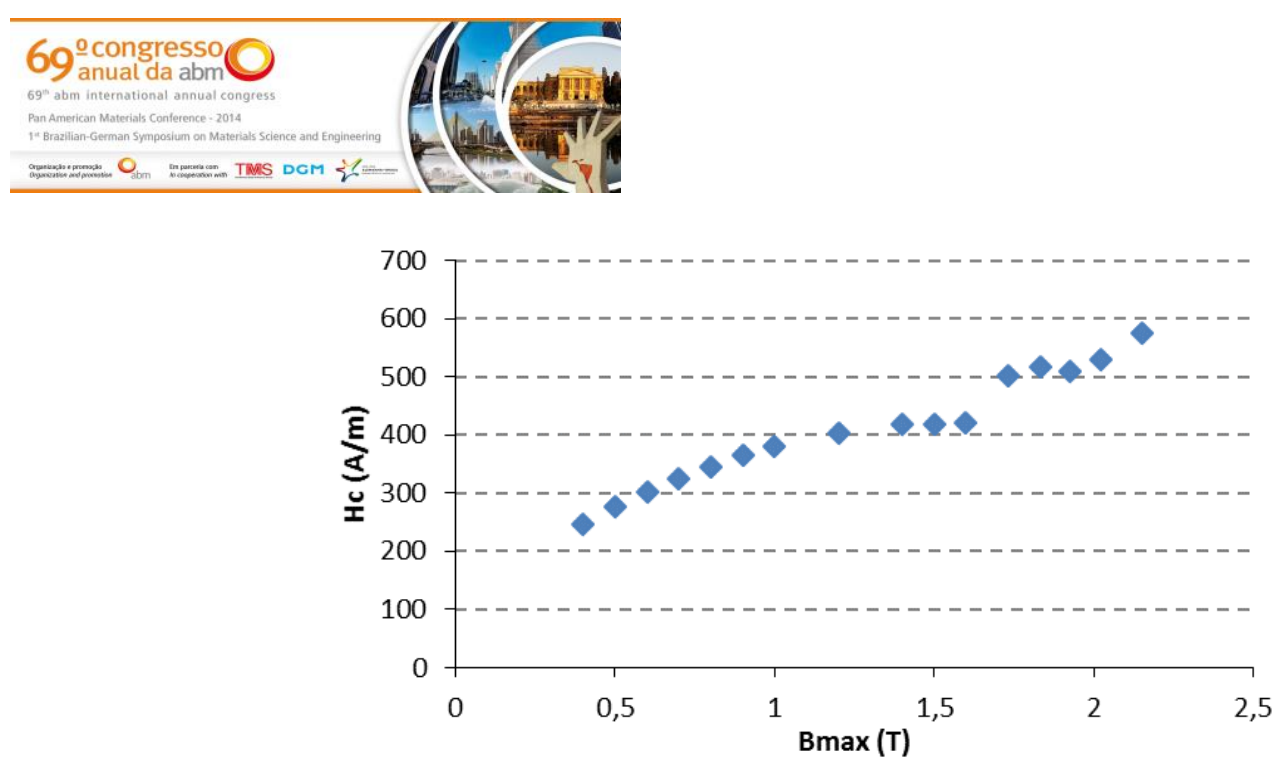

Figura 6: Evolução do campo coercivo em função do aumento da indução máxima.

\section{CONCLUSÕES}

Os resultados aqui apresentados mostram que a energia dissipada por histerese se relaciona com a indução máxima de acordo com uma lei de potencia. No entanto, o valor do expoente foge do previsto pela lei de Steinmetz para o intervalo de dados entre as induções 1,2 e 1,6 T. Tal fato pode ser explicado pelo comportamento do campo coercivo que apresente valor estável nesse intervalo de induções, porém, retoma o crescimento para valore de $B_{\max }$ superiores a 1,7 T. Uma provável causa para esse acontecimento pode ser atribuída ao método de extração dos ciclos de histerese para os maiores valores de indução máxima avaliados.

\section{Agradecimentos}

Os autores agradecem à Brasmetal Waelzholz $\mathrm{SA}$, que gentilmente cedeu o material para esse trabalho, ao Instituto de Física e ao Instituto de Pesquisas Tecnológicas IPT. D. L. Rodrigues-Jr e F. J. G. Landgraf agradecem à Coordenação de Aperfeiçoamento de Pessoal de Nível Superior - CAPES pelo suporte financeiro.

\section{REFERÊNCIAS}

1 Bertotti G. Connection between microstructure and magnetic properties of soft magnetic materials. Journal of Magnetism and Magnetic Materials. 2008;320:2436-42.

2 de Campos MF, Yonamine T, Fukuhara M, Landgraf FJG, Achete CA, Missell FP. Effect of Frequency on the Iron Losses of $0.5 \%$ and $1.5 \%$ Si Nonoriented Electrical Steels. IEEE Transactions on Magnetics. 2006;42(10).

3 Steinmetz CP. On the law of hysteresis. American Institute of Elecfrical Engineers Transactions. 1892;9:344.

4 Landgraf FJG, M. de Campos EMF. On the Steinmetz hysteresis law. Journal of Magnetism and Magnetic Materials. 2008;320:531-534.

5 Foner S. Versatile and Sensitive Vibrating-Sample Magnetometer. The Review of Scientific Instruments. 1959;July(7):548-557.

6 Cullity BD, Graham CD. Introduction to magnetic Materials. 2 ${ }^{\underline{a}}$ ed. Piscataway: IEEE Press; 2009.

7 Ball JD. Investigation of magnetic laws for steel and other materials. Journal of the Franklin Institute. 1916;4(181):459-504

8 Landgraf FJG, Campos MF, Leicht J. Hysteresis loss subdvision. Journal of Magnetism and Magnetic Materials. 2008;320:2494-98.

* Contribuição técnica ao 69ำ Congresso Anual da ABM - Internacional e ao 14ํㅡㄹ ENEMET - Encontro Nacional de Estudantes de Engenharia Metalúrgica, de Materiais e de Minas, 21 a 25 de julho de 2014, São Paulo, SP, Brasil. 